\title{
A Simulation-Based Performance Analysis of a Cluster-based Routing Scheme for Wireless Mesh Networks
}

\author{
Madhusudan Singh ${ }^{1}$, Song-Gon Lee ${ }^{2, \dagger}$ and Dhananjay Singh ${ }^{3}$ \\ ${ }^{1}$ Samsung Display, Yongin-si, Gyeonggi-do, 446-711, Korea \\ ${ }^{2}$ Division of Computer \& Information Engineering, Dongseo University \\ Busan, 617-716, Korea \\ ${ }^{3}$ Department of Electronics Engineering, Hankuk University of Foreign, \\ Yongin-si, Gyeonggi-do, 449-791, Korea \\ ${ }^{1}$ sonu.dsu@gmail.com, ${ }^{2}$ nok60@dongseo.ac.kr, ${ }^{3}$ dan.usn@gmail.com \\ ${ }^{\dagger}$ The corresponding author
}

\begin{abstract}
The cluster-based routing protocol $(C B R P)$ is a grouping mechanism that supports unicast and multicast path discovery for low congestion and packet loss in routing mechanisms for multi-hop wireless mesh networks. No simulation analyses or comparisons with other routing protocols have been reported. In this paper, we have evaluated the performance of the CBRP and compared it to AODV and OLSR through software simulations. The scheme indicates several better performance levels in terms of the channel capacity, path discovery, packet delivery ratio, and end-to-end delay, at the sacrifice of routing overhead degradation.
\end{abstract}

Keywords: Wireless Mesh Networks, AODV, OLSR, CBRP

\section{Introduction}

Wireless mesh networks (WMNs) are emerging as a low cost and flexible wireless network technology that has a self-organizing infrastructure. WMNs are flexible, cost effective, and have light infrastructures based on wireless technology. Industry and academia place very high demands on WMNs because of their advantages, such as the network range extension, resilience, fault tolerance and high bandwidth capabilities. Wireless mesh networks extend the coverage of wireless local area network (WLAN) technology to a wider area with multihop routing functionality [1]. WLAN technology has a standard name of IEEE 802.11, and wireless mesh networks are known as IEEE 802.11s. WMNs are the latest technology that has a substantial amount in common with mobile ad hoc network (MANET) [2].

The architecture of WMNs has been classified into three categories based on the node functionality in the networks:

Infrastructure WMNs,

Infrastructure-less WMNs,

Hybrid WMNs.

This paper is a revised and expanded version of a paper entitled [Cluster-based routing scheme for Wireless Mesh Networks] presented at [The 13th International Conference on Advanced Communication Technology (ICACT), Gangwon-Do, South Korea, 2011]. 
In infrastructure WMNs, mesh routers provide connectivity to mesh nodes in the infrastructure form. In infrastructure-less WMNs, nodes make the mesh networks and perform mesh functions, such as routing and self-configuration. Hybrid WMNs are a combination of infrastructure and infrastructure-less WMNs [3-4]. In this type of network, mesh clients access the networks via mesh routers and other mesh clients. The current infrastructure WMN is the most popular network because of its stability and management ease.

WMNs are associated with many research fields, one of which concerns routing. Many routing protocols have been proposed, and a substantial amount of work has been performed by researchers; however, much more research could be conducted in this field. The exiting routing protocol has path discovery with the help of a broadcast method [5]. For this reason, a node requires a substantial amount of power, and there are message collisions when congestion occurs in networks. Toward addressing these concerns, one report [6] proposed a cluster-based routing protocol (CBRP) in which all of the nodes in mesh networks were grouped into different clusters, and each cluster has a cluster head $(\mathrm{CH})$. Because this protocol sets up a path between nodes using unicast and multicast methods, the networks produce less congestion and data loss. In a previous work [6], only one protocol was proposed, and some expected effects were analyzed heuristically. This approach requires further performance investigation.

In this paper, we present further research on the CBRP. We defined a pseudo-code for the CBRP and conducted a performance analysis in a simulation environment. We made a performance comparison with Ad hoc On-Demand Distance Vector (AODV) routing [7] and Optimized Link State Routing (OLSR) [8] in terms of several performance metrics, such as channel capacity, hop count, packet delivery ratio, end-to-end delay, and routing overhead. In Chapter 2, we briefly introduce some background knowledge on the existing routing protocols for WMNs and explain the CBRP. In Chapter 3, we describe the pseudo-code for the CBRP. In Chapter 4, simulation results and performance analyses are given. Finally, in Chapter 5, conclusions are described.

\section{Existing routing protocol and the CBRP for WMNs}

\subsection{The existing routing protocol}

International organizations are actively providing specifications for mesh networks, such as IEEE802.11, IEEE802.15, and IEEE802.16 [9]. WMNs can easily maintain networks by adding and deleting nodes over the networks. In this article, we are concentrating on routing protocols of IEEE802.11s networks.

Many researchers have proposed a number of routing techniques for WMNs. Many ondemand routing protocols, such as Dynamic Source Routing (DSR) [10] and AODV, have been presented for wireless networks. These protocols are traditional routing protocols for MANET [11]. This type of routing protocol uses a broadcast method for path setup and communication. After these traditional protocols, researchers propose opportunistic routing protocols [12], such as extremely opportunistic routing (ExOR) [13], resilient opportunistic mesh routing (ROMER) [14], and the routing protocol of Zhong et al. [15], which are also broadcast natured. The existing routing protocols use a broadcast message in an initial stage. Although they have an ability to avoid duplicate transmissions, these protocols consume a substantial amount of energy and induce collisions.

In this paper, we propose an alternative approach, which is a novel cluster-based routing protocol for wireless mesh networks. We provide extra power and responsibility to the mesh portal point (MPP) and the cluster head of each group. 


\subsection{The cluster-based routing protocol}

In our previous work [6], we proposed a cluster-based routing protocol for WMNs. With this protocol, we provided the solution of the above-mentioned problem regarding existing routing protocols. If networks are partitioned into clusters, then we can reduce the initial broadcast to all of the nodes. Because each cluster has one head that has all of the information on its neighbors, the path request is multicast to different cluster heads only. In this scheme, we divide the mesh networks into groups (Figure 1). The MPP assigns one node to be a cluster head $(\mathrm{CH})$ of each cluster group and stores the cluster head information in its own table, such as $\mathrm{CH}$ and $\mathrm{CH}$ neighbors. Figure 1 presents cluster groups in WMNs.

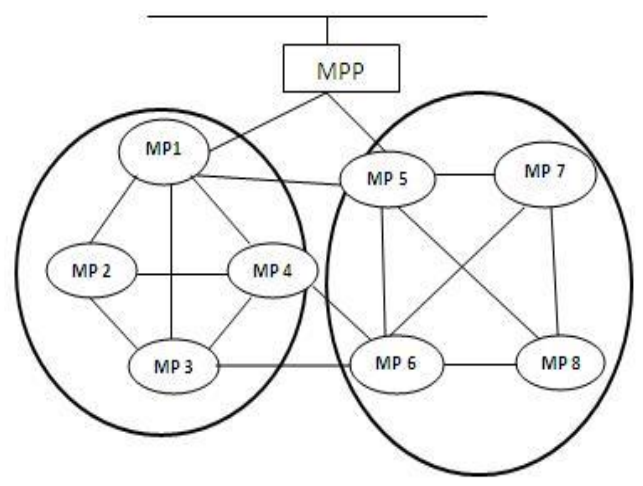

Figure 1. Wireless mesh networks divided into cluster groups

In our routing protocol, the path establishment process is comprised of 4 steps: setup of the cluster head, path creation, path reply, and path setup. The details of the process are as follows.

1) Setup of Cluster Head.

Each cluster head has extra authority compared to other cluster members. Each cluster head has two tables. One table stores information on neighbor cluster heads, and the second table stores information about cluster group members, which is assigned by the MPP. Every cluster member stores the information on its $\mathrm{CH}$.

2) Path creation.

When a normal cluster member wants to communicate with a destination node, it sends a path request (PREQ) message to its cluster head (Figure 2(a)). Then, the cluster head checks its own group member list. If a destination exists in the same group, it quickly sends a path reply with path information, and a source node starts a transmission according to that path. If a destination node belongs to another cluster, the cluster head sends a PREQ message to the mesh portal (Figure 2(b)), and the mesh portal multicasts a PREQ message to all of the cluster heads (Figure 2(c)). Cluster heads then check their own group table, and if any $\mathrm{CH}$ finds a destination node in an area, it sends a PREQ message to the destination node (Figure 2(d)). The destination node sends its own status to its cluster head (Figure 2(e)).

3) Path reply.

After receiving the status message from the destination node, the destination cluster head sends a path reply message to the mesh portal (Figure 3(f)). This reply message is intended to be sent to the source node with the destination path information. MPP forwards the PREP, 
which is received from destination $\mathrm{CH} 2$ to source $\mathrm{CH} 1$, as shown in Figure $3(\mathrm{~g})$. $\mathrm{CH} 1$ forwards it to the source node.

4) Path setup.

A bidirectional path established between the $\mathrm{CH} 1$ and $\mathrm{CH} 2$ nodes is shown in (Figure 3(h)), and $\mathrm{CH} 1$ forwards the destination information to the source node (Figure 3(i)). Thus, the final path between the source and destination nodes is established, as shown in Figure $3(\mathrm{j})$.

In this scheme, MPP multicasts during path discovery only once, and the remaining transmission uses unicast messaging. Hence, this scheme reduces the power consumption and improves the network performance at the mesh portal. The cluster head periodically updates its own table, which helps to detect any change that occurs in networks. Furthermore, in the case of link-failure retransmits to the mesh portal by the $\mathrm{CH}$, error message (RERR) broadcasting is eliminated. However, when the cluster head fails, then broadcasting is required.
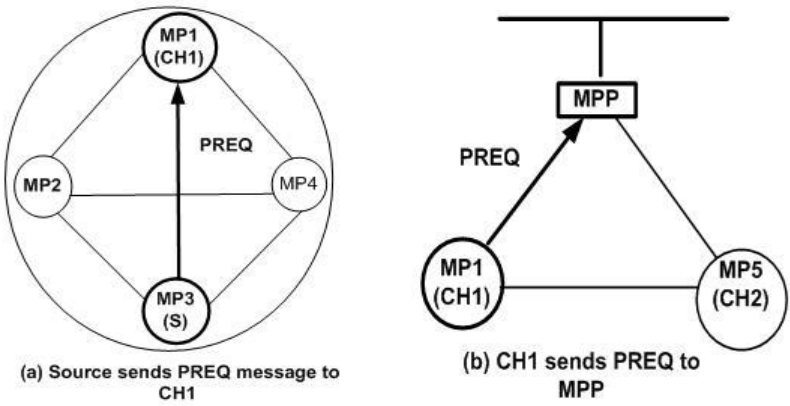

(b) $\mathrm{CH} 1$ sends PREQ to MPP

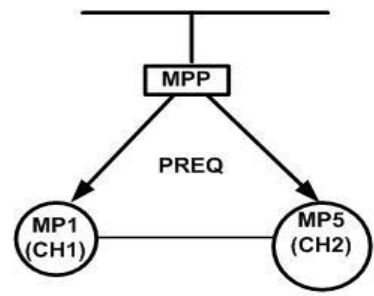

(c) MPP multicast PPREQ to

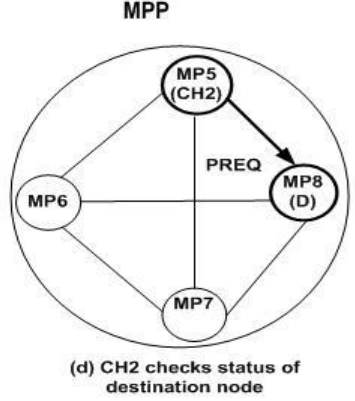

$\mathrm{CHs}$

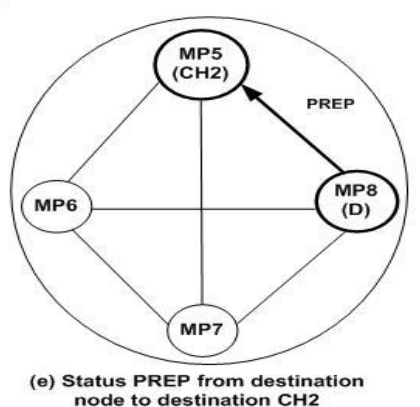

Figure 2. Path request (PREQ) process in the CBRP 


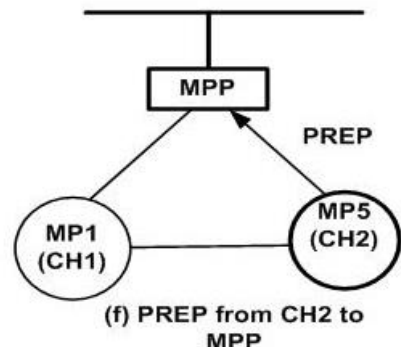

MPP

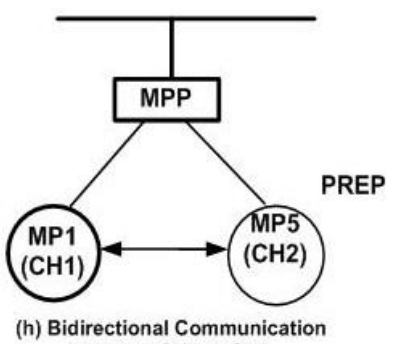
between $\mathrm{CH} 1$ to $\mathrm{CH} 2$

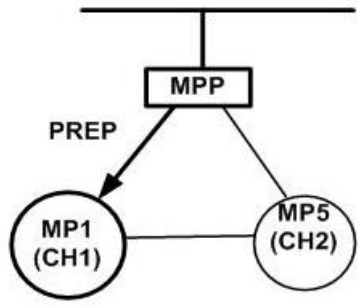

(g) PREP from MPP to $\mathrm{CH} 1$

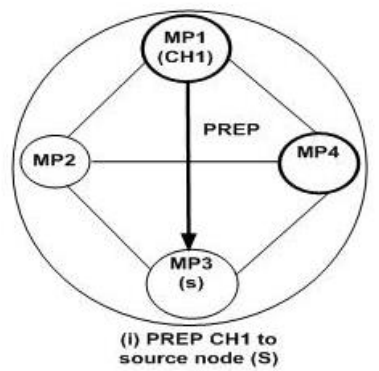

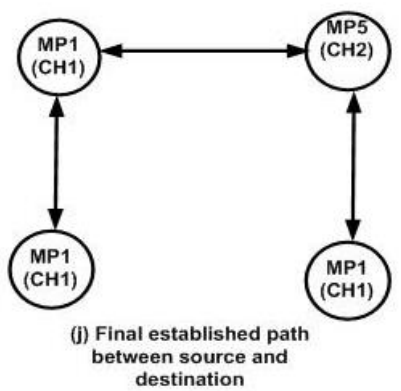

Figure 3. Path reply (PREP) process in the CBRP

\section{Pseudo-code for the CBRP}

\subsection{The Basic Process of CBRP}

There are 5 main basic processes of the CBRP, as follows:

(a) Partitioning of the network graph,

(b) Distribution of cluster connectivity messages to $\mathrm{CH}$,

(c) Distribution of routing information to the individual nodes by each $\mathrm{CH}$,

(d) Intra cluster route made via MPP,

(e) Inter cluster route made through the calculation of neighbors between the source and the destination.

We explain our method with the aid of pseudo-code. In pseudo-code, $\mathrm{CH}$ is the symbol for cluster head, and S and D represent for the source and destination, respectively.

1) Route Assignment Process.

(1) Intra cluster route:

(a) $\mathrm{R}=$ Rreq+Rrep, 
(b) $\mathrm{Req}=(\mathrm{S}+\mathrm{CHs})+(\mathrm{CHs}+\mathrm{MPP})+(\mathrm{MPP}+\mathrm{CHd})+(\mathrm{CHd}+\mathrm{D})$,

(c) $\mathrm{Rrep}=(\mathrm{D}+\mathrm{CHd})+(\mathrm{CHd}+\mathrm{MPP})+(\mathrm{MPP}+\mathrm{CHs})+(\mathrm{CHs}+\mathrm{S})$,

where $\mathrm{R}=$ route, Rreq=route request, Rrep=router reply, $\mathrm{S}=$ source, $\mathrm{CHs}=$ source cluster head, $\mathrm{CHd}=$ destination cluster head, MPP=mesh portal point, and $\mathrm{D}=$ destination.

(2) Inter cluster route:

(d) $\mathrm{R}=$ Rreq+Rrep,

(e) $\mathrm{Rreq}=\mathrm{S}+\mathrm{CHs}$,

(f) $\mathrm{Rrep}=\mathrm{CHs}+\mathrm{S}$.

(3) Pseudo-code for inter cluster.

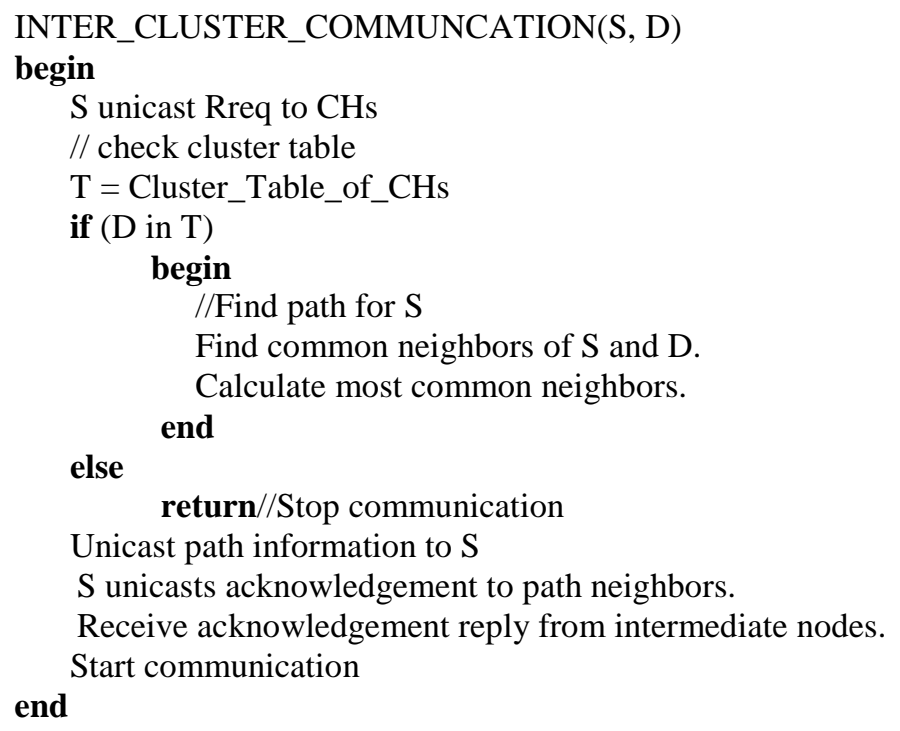

(4) Pseudo-code for intra cluster Group:

\section{INTRA_CLUSTER_GROUP(S, D)}

begin

$\mathrm{S}$ unicast to CHs.

// CHs review table.

$\mathrm{T}=$ cluster_table_of_CHs

if $(S$ in $T$ and $D$ in $T$ )

begin

INTER_CLUSTER_COMMUNCATION(S, D)

$$
\text { end }
$$

else

Unicast RREQ to MPP.

MPP multicast to all $\mathrm{CHs}$

for each $\mathrm{CH}$ do

begin

$\mathrm{CH}$ checks its own Table

CHd finds destination node

// Chd checks availability of D 


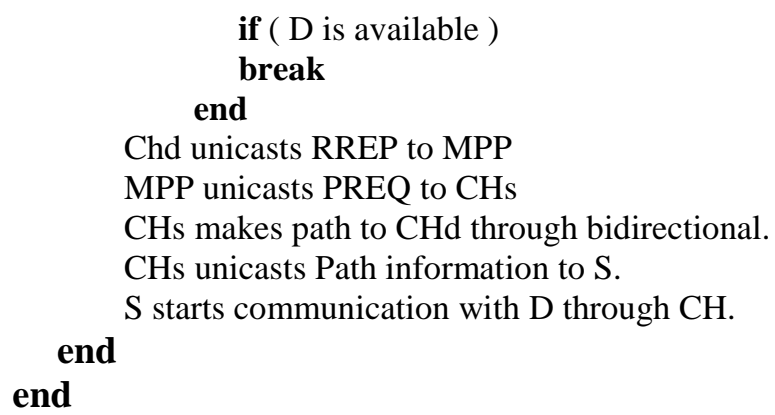

\section{Performance analysis of the CBRP}

In a WMN environment, we analyzed the performance of the CBRP protocol by performing simulations with OPNET. Table 1 shows the parameters for the simulation in the OPNET simulator. We selected AODV and OLSR for comparing targets because they are typical routing protocols for WMN that work in a reactive and proactive manner, respectively $[7,8]$.

A different simulation scenario has been studied according to 5 different performance metrics: channel capacity, path discovery, packet delivery ratio, end-to-end delay and routing overhead. The WMN nodes are randomly placed. To analyze the channel capacity of WMNs, ten cluster heads were placed in a $1000 \times 1000 \mathrm{~m}^{2}$ area. The packet size was set to 512 bytes, and the packet transmission rate was set to 10 packets/sec. The radio range was set to $50 \mathrm{~m}$. The radio bandwidth is $2 \mathrm{Mbps}$. The total number of nodes is 100 .

Table 1. Parameters common to all simulations

\begin{tabular}{|c|c|}
\hline Parameter & Value \\
\hline Simulation area & $1000 * 1000 \mathrm{~m}^{2}$ \\
\hline Total number of nodes & 100 \\
\hline Total number of CHs & 10 \\
\hline Simulation Time & $200 \mathrm{~s}$ \\
\hline Packet size & $512 \mathrm{bytes}$ \\
\hline Bandwidth & $2 \mathrm{Mbps}$ \\
\hline Packet rate & $10 \mathrm{pkts} / \mathrm{s}$ \\
\hline
\end{tabular}

To evaluate the performance of the CBRP mechanism, we must analyze the capacity of each $\mathrm{CH}$ and the capacity of the whole network. We also used a single radio system for wireless mesh networks, which indicates that the same radio is used for both access and wireless backhaul. This option represents the lowest cost entry point in the deployment of a wireless mesh network infrastructure. The actual average channel capacity of a mesh node is between $1 / \mathrm{N}$ times the channel capacity and $(1 / 2)^{N}$ times the channel capacity, where $\mathrm{N}$ is the number of wireless hops in the longest path between a wireless single node and wired networks [16].

\subsection{Channel capacity of the CBRP}

The channel capacity of each cluster head is measured by the average data transmission rate for each cluster head. The total channel capacity is measured by summing the data transmission rate of all cluster head. 
Figure 4 shows the channel capacity performance comparison of each node with respect to existing routing protocols AODV, OLSR and CBRP. When the number of nodes is 1, then all three routing protocols have the same channel capacity; however, when the number of nodes in the network is increased, the routing protocols' capacity is decreased because of network congestion and collision. However, the CBRP still has a better performance compared to the AODV and OLSR. When the number of $\mathrm{CHs}$ is 3, the largest difference occurs between the CBRP and AODV, which is almost $6 \mathrm{Mbps}$. The AODV uses a broadcast method for communication that requires a substantial amount of traffic. However, the CBRP and OLSR use the unicast method for communication, which is why their capacity performance is better than AODV. Although the OLSR and CBRP use unicast methods, the CBRP shows better performance than the OLSR. The OLSR is not supportive for large-scale networks.

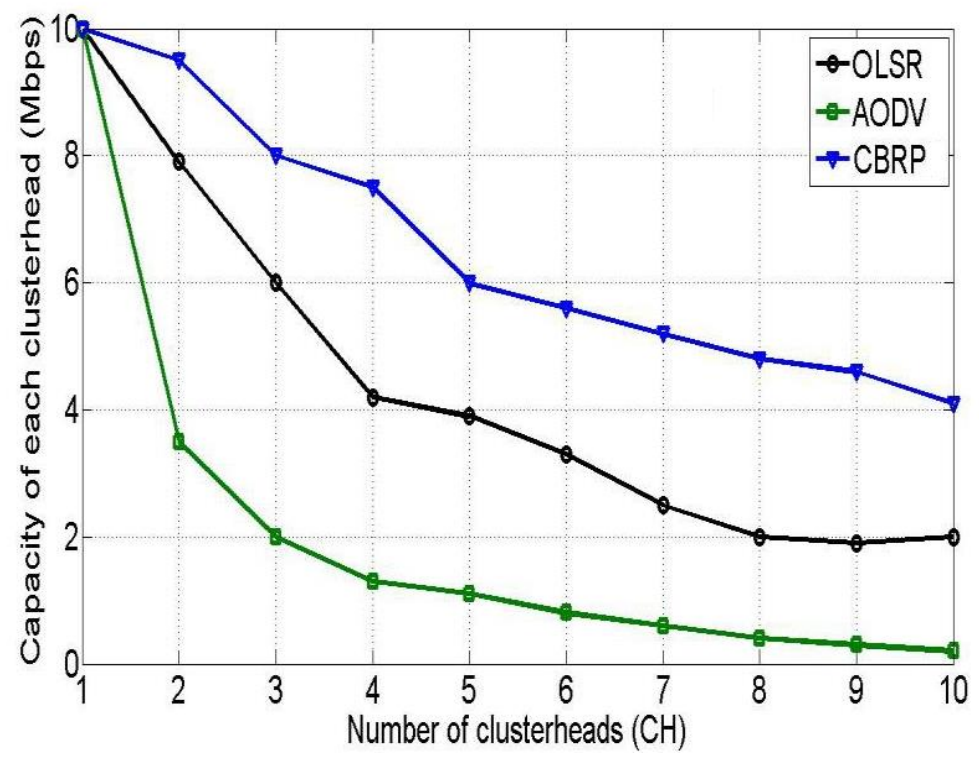

Figure 4. Comparison of each $\mathrm{CH}$ capacity

The simulation result in Figure 5 presents a comparison of the total channel capacity of the CBRP and the routing protocols AODV and OLSR. When the number of nodes is 1 , then all three routing protocols have the same channel capacity. However, as the number of $\mathrm{CHs}$ increased, the AODV performance decreased but the capacities of the OLSR and CBRP increased. In the figure, for up to 4 nodes, the CBRP's performance is less than that of the OLSR's; however, for 5 nodes and higher, the OLSR's performance decreases compared to the CBRP. After 4 nodes, the OLSR and AODV saturate to the values $6 \mathrm{Mbps}$ and $28 \mathrm{Mbps}$, respectively, but the CBRP still continues an increasing trend. In a large-scale network, the CBRP provides a better capacity performance when compared to the OLSR and AODV. 


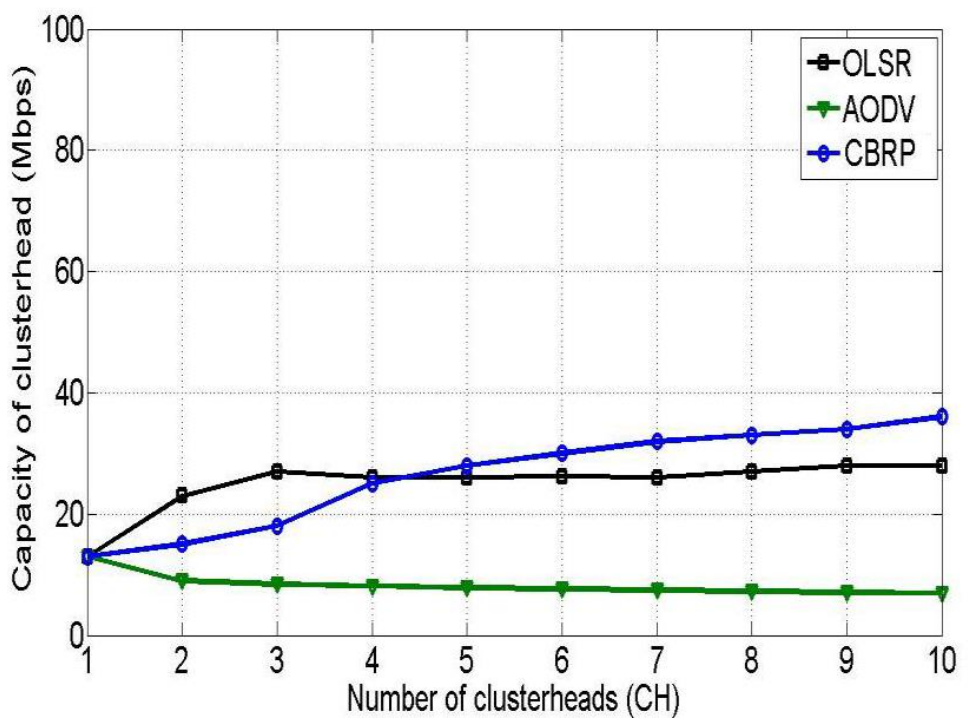

Figure 5. Comparison of the $\mathrm{CH}$ capacity in the whole WMN

\subsection{Number of Paths discovered}

Figure 6 represents the average path that is discovered in the CBRP, AODV and OLSR routing protocols. While the AODV can discover the highest number of paths, the OLSR and CBRP discover a smaller number because of their proactive mechanism. The AODV uses a broadcast message, and the OLSR has a predefined path discovery method. However, the CBRP routing uses a unicast and multicast method for path discovery. For this reason, it has a lower number of path discovery features. Each routing protocol has its own pros and cons, depending on the network conditions and requirements.

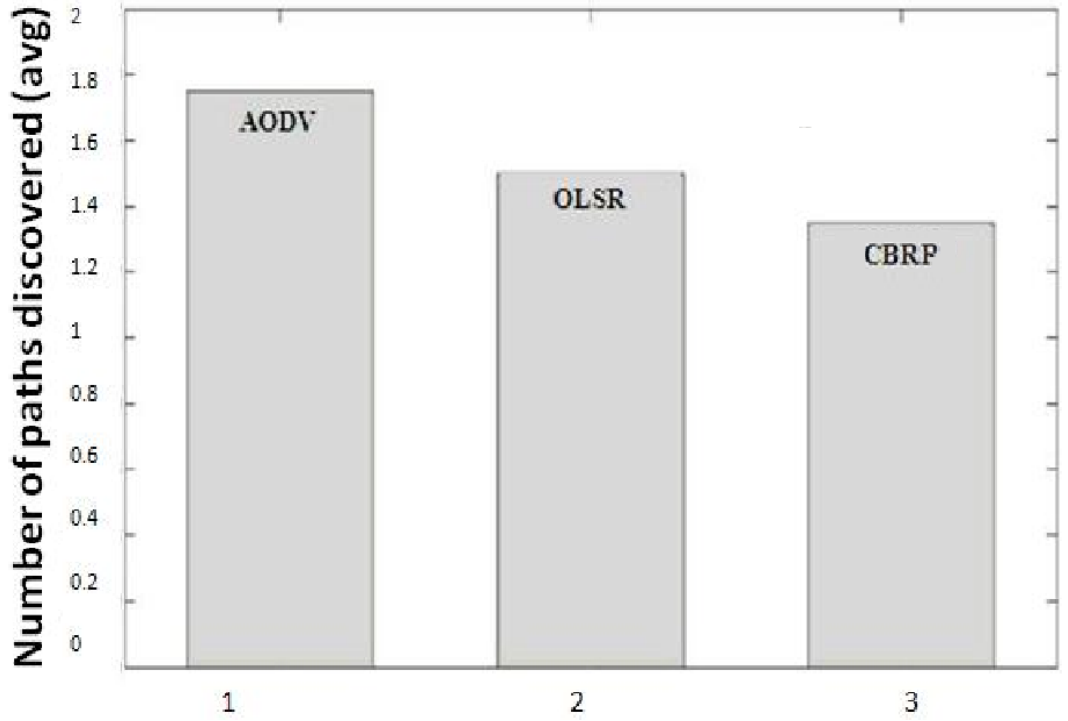

Figure 6. Average numbers of paths discovered by each protocol. 


\subsection{Packet delivery ratio}

The packet delivery ratio (PDR) denotes the ratio of the total packets received by the destination to the total packets sent by the source during the whole simulation, which reflects the routing efficiency. For every 1 second, we measured the number of packet that have been sent and received during 200 second. The PDR is used to determine the optimal number of delivery paths under different circumstances and is also used to determine the suitability of different clustering algorithms for supporting multi-path routing. The more packets are received, the better the performance is.

In other words,

$$
P D R=\frac{\sum P r_{i}}{\sum P s_{i}},
$$

where $P D R$ denotes the packet delivery ratio during the whole simulation, $\operatorname{Pr}_{i}$ denotes the number of packets received during the $i$-th time frame by the destination, and $P s_{i}$ denotes the number of packets sent during the $i$-th time frame by the source.

Figure 7 shows the PDR for the AODV, OLSR and CBRP with respect to the number of nodes. The CBRP performs better than the OLSR and AODV for a high and low network size. The AODV performs better than the OLSR in terms of the number of nodes. CBRP delivers almost 40 60\% packets in 20 to 100 nodes. Because CBRP's $40 \%$ performance is much better that OLSR's $20 \%$ and AODV's $28 \%$ even when number of node is 100 , our protocol is better than the other in a large network.

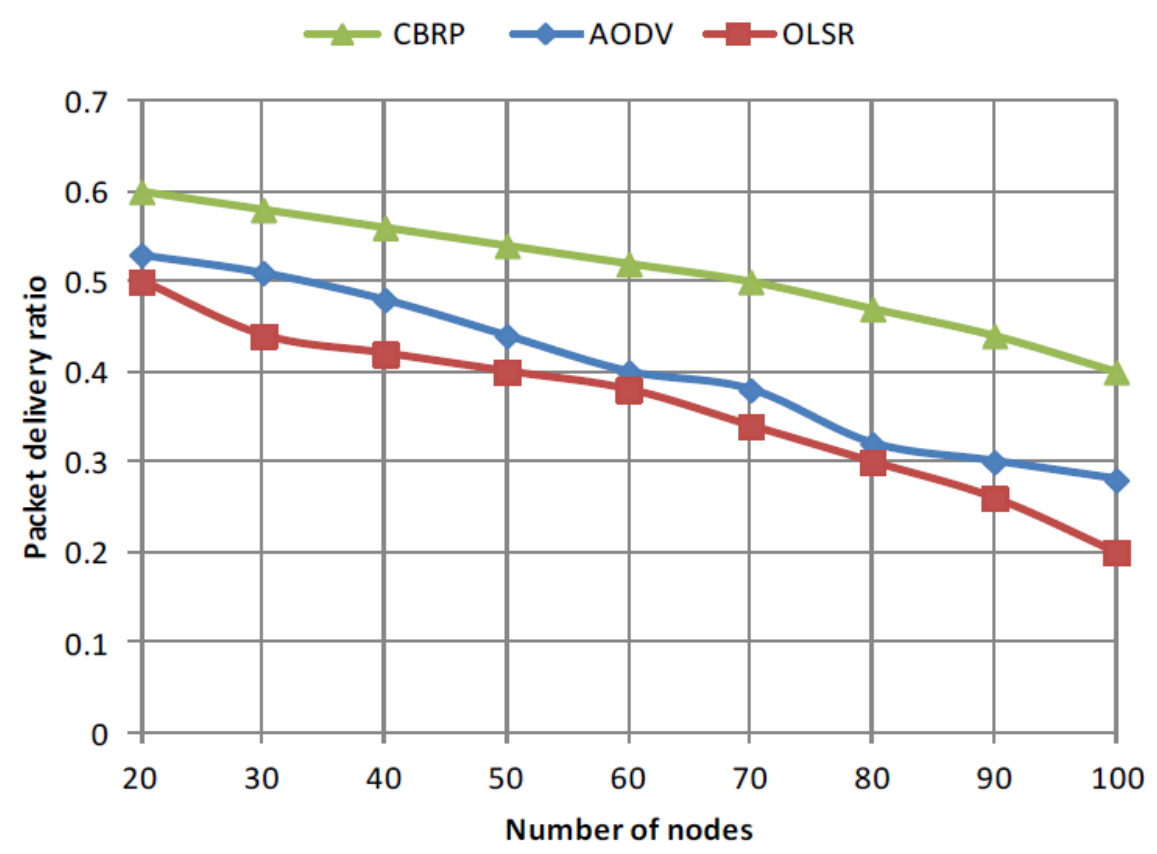

Figure 7. The packet delivery ratios as a function of the number of nodes 


\subsection{End-to-end (ETE) delay}

The data transmission time from a source to a destination is known as the "end-to-end (ETE) delay' and includes the delay time induced by the cache during the discovery phase, the queue waiting time, and the transmission time over the links. For every $1 \mathrm{sec}$ during 200 seconds, we measured the number of packet that have been sent and the time difference between sender and receiver.

A lower ETE delay signifies a better performance. The ETE delay is defined as follows:

$$
\text { ETE delay }=\frac{\Sigma\left(T_{R_{i}}-T_{S_{i}}\right)}{\Sigma N_{P_{i}}}
$$

where $T_{R i}$ denotes the time of receiving packets, $T_{S_{i}}$ represents the time of sending packets, and $N_{p i}$ denotes the number of packets at the $i$-th time frame. The delay time increases as the number of nodes increases.

In Figure 8, we can see that the CBRP has a low ETE delay when compared to the OLSR and AODV. The OLSR and AODV almost have more than a 2 sec delay time, but for the CBRP, the delay is $<1.5 \mathrm{sec}$ at 100 nodes. The average ETE delay difference between the CBRP and AODV is almost 1.2 seconds, and between the CBRP and OLSL it is almost 0.6 seconds. These results ensure better performance for the CBRP when compared to the AODV and OLSR.

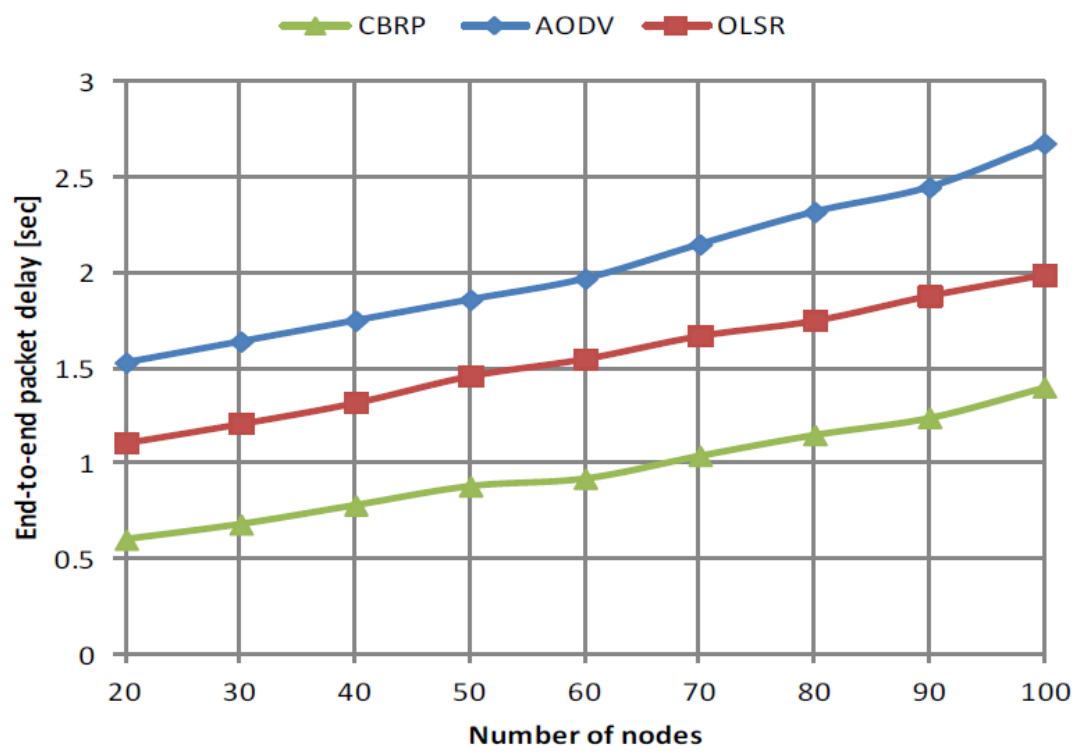

Figure 8. The end-to-end delay as a function of the number of nodes 


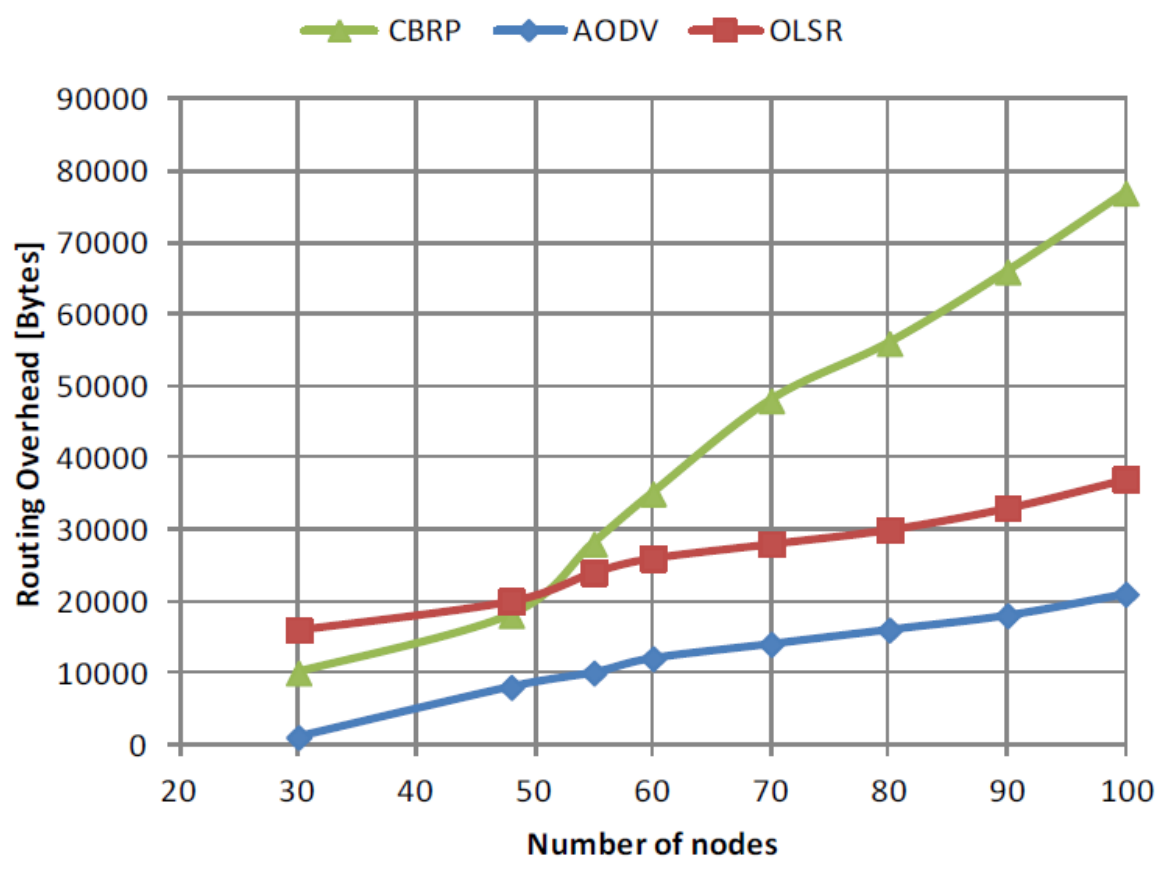

Figure 9. The routing overhead as a function of the number of nodes

\subsection{Routing Overhead}

Routing overhead is the total number of routing packets that are transmitted over a network during a simulation time of $200 \mathrm{~s}$. Figure 9 indicates that the routing overhead of the CBRP is low when compared to the OLSR for $\leq 50$ nodes. After 50 nodes, the routing time rises very quickly because of node congestion and traffic collisions. This result is a drawback of our previously proposed routing protocol. The AODV and OLSR have less overhead when compared to the CBRP. When the number of nodes is less, then the CBRP provides a better performance compared to the OLSR. When the number of nodes is increased, the collision and congestion occurrences also increase in the network because of overhead. The CBRP has almost an additional 50,000 bytes of overhead when compared to the OLSR. The CBRP has almost an additional 42,000 bytes of overhead when compared to the OLSR at 100 nodes.

We have compared the performance of the CBRP with respect to the AODV and OLSR. With the exception of the routing overhead, the CBRP outperforms the AODV and OLSR for the channel capacity, path discovery, packet delivery ratio, and end-to-end delay. Thus, the CBRP outperforms the other protocols at the sacrifice of routing overhead degradation.

\section{Conclusions}

In this paper, we analyzed the performance of a cluster-based routing protocol for wireless mesh networks. This study presented further research for the routing scheme that was proposed earlier by our research team.

The proposed routing scheme divides the network into a number of groups (clusters). If the source and destination are in the same cluster, then the cluster head sends path information to the source as a unicasting message. If the destination node is not a member of the same cluster as a source, then the cluster head sends a route request message to the MPP. The MPP forwards this route request to all of the cluster heads in the form of a multicast message. As a 
result, it again does not require the broadcasting of the route request for the path. However, in case of an existing routing protocol, flooding takes place over the whole network even when the destination is very near to the source. As a result, this approach appears to be very costly. As the group size is increased, fewer multicasts and more unicasts exist. However, an overload on the cluster head can occur. A decision on the size of the group and the number of clusters depends on the topology and application of the networks.

Through software simulation, we have evaluated the performance of the proposed CBRP compared to the AODV and OLSR. The scheme shows several better performances in terms of the channel capacity, path discovery, packet delivery ratio, and end-to-end delay at the sacrifice of routing overhead degradation.

A possible future research direction would be to determine the group size and the number of clusters, depending on the topology and application of the networks.

\section{Acknowledgement}

This work was supported by Basic Science Research Program though the National Research Foundation of Korea (NRF) funded by the Ministry of Education, Science and Technology (Grant number: 2012-0002273 ).

\section{References}

[1] I. F. Akyildiz, X. Wang and W. Wang, "Wireless mesh networks: a survey”, Computer Networks, vol. 47, (2005), pp. 445-487.

[2] IEEE P802.11s task Group IEEE Unapproved draft standard P802.11s/D4.0, (2010) December.

[3] Institute of Electrical and Electronics Engineers, "802.11s Proposal - Joint SEEMesh/ Wi- Mesh Proposal to 802.11 TGs", in IEEE 802.11-06/0328r0, (2006).

[4] Z. Yan, L. Jijun and H. Honglin, "Wireless Mesh Networking, Architectures Protocols and Standard", USA: Auerbach Publications, (2007).

[5] M. S. Kuran and T. Tugcu, "A survey on emerging broadband wireless access technologies", Computer Networks, vol. 51, (2007), pp. 3013-3046.

[6] S. Madhusudan, S. -g. Lee, T. W. Kit and L. J. Huy, "Cluster-based routing scheme for Wireless Mesh Networks", Proceeding of 2011 13th International Conference on Advanced Communication Technology (ICACT), (2011), Gangwon-Do, South Korea.

[7] B. -N. Cheng, M. Yuksel and S. Kalyanaraman, "Orthogonal Rendezvous Routing Protocol for Wireless Mesh Networks”, IEEE/ACM Transactions on Networking, vol. 17, (2008), pp. 542-555.

[8] T. Clausen and P. Jacquet, "Optimized Link State Routing Protocol (OLSR)", RFC 3626, (2003).

[9] M. E. M. Campista, P. M. Esposito, I. M. Moraes, L. H. M. K. Costa, O. C. M. B. Duarte, D. G. Passos, C. V. N. Albuquerque, D. C. Muchaluat-Saade and M. G. Rubinstein, "Routing metrics and protocols for wireless mesh networks," IEEE Network, vol. 22, (2008), pp. 6-12.

[10] S. Waharte, R. Boutaba, Y. Iraqi and B. Ishibashi, "Routing protocols in wireless mesh networks: challenges and design considerations", Multimedia Tools Appl, vol. 29, (2006), pp. 285-303.

[11] R. O. Schoeneich and M. Golański, "Mesh Cluster Based Routing Protocol: Enhancing Multi-hop Internet Access using Cluster paradigm", Proceeding of EUROCON 2007 The IEEE International Conference on "Computer as a Tool", (2007), Warsaw, Poland.

[12] N. M. AL-Kharasani and Z. A. Zukarnain, "Performance Evaluation of Routing with Load-Balancing in Multi-Radio Wireless Mesh Networks", JDCTA: International Journal of Digital Content Technology and its Applications, vol. 5, (2011), pp. 64-71.

[13] S. Biswas and R. Morris, "ExOR: Opportunistic Routing in Multi-hop Wireless Networks", Proceeding of ACM SIGGCOMM'05, (2005), Philadelphia, Pennsylvania, USA.

[14] Y. Yuan, H. Yuan, S. H. Wong, S. Lu and W. Arbaugh, "ROMER: resilient opportunistic mesh routing for wireless mesh networks", Proceeding of the 1st IEEE Workshop on Wireless Mesh Networks, (2005), Santa Clara, California, USA.

[15] Z. F. Zhong and S. Nelakuditi, "On the efficacy of opportunistic routing", Proceeding of the IEEE SECON'07, (2007), San Diego, California, USA.

[16] Belair Networks, "Capacity of Wireless Mesh Networks" white paper, BDMC00040-C02, (2006). 


\section{Authors}

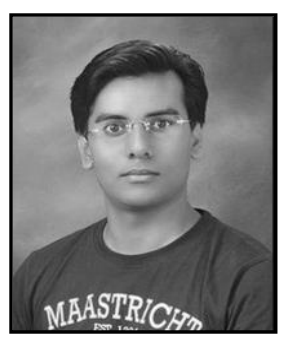

Madhusudan Singh received his Ph.D. degree in the Dept. of Ubiquitous IT, from Dongseo University (DSU), Busan, South Korea in 2012. M. Tech. degree in Dept. of IT with spec. in Software Engineering from Indian Institute of Information Technology, Allahabad (IIIT-A), India in 2008 and his Master degree in the Dept. of Computer Application from UP Technical University(UPTU), Lucknow, India in 2006. Bachelor degree is the Dept. of Computer Applications from VBS Purvanchal University, Jaunpur, India. Dr. Singh was a visiting research scholar at University of the Pisa, Italy in 2010. Currently, he is a senior engineer, at Samsung Research Group at Samsung Display, South Korea. His fields of research interests are Wireless Mesh Networks, Information Security, Wireless Sensor Networks, Signal System, Image Processing, and Software Engineering.

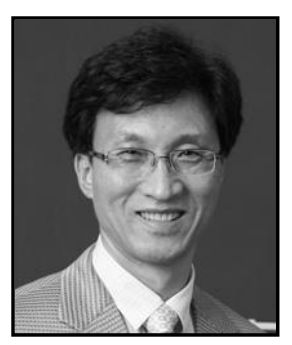

Sang-Gon Lee received his BEng, MEng, and $\mathrm{PhD}$ degree in electronics engineering from Kyungpook National University, Korea, in 1986, 1988, and 1993, respectively. He is a professor at the Division of Computer \& Information Engineering, Dongseo University. He was a visiting scholar at Queensland University of Technology, Australia from August 2003 to July 2004 and at University of Alabama in Huntsville from July 2012 to Jun 2013. His research areas include information security, network security, wireless mesh/sensor network and future internet.

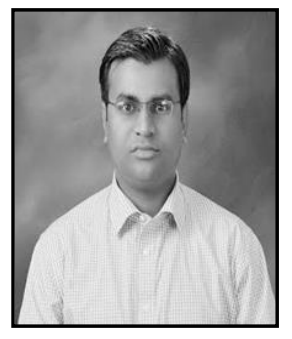

Dhananjay Singh received his B. Tech. in the Dept. of Computer Science and Engineering from United College of Engineering and Research (UCER), M. Tech. in Dept. of IT with specialization in Wireless Communication and Computing from Indian Institute of Information Technology-Allahabad (IIIT-A), India (2003 \& 2006) and $\mathrm{PhD}$ degree in Dept. of Ubiquitous-IT at Dongseo University(2010). Currently, he is assistant professor in the Dept. of Electronics Engineering at Hankuk University of Foreign Studies (HUFS), Yongin-si, Korea. He had worked with Future Internet Research Team at Electronics Telecommunication Research Institute (ETRI) and National Institute Mathematical \& Sciences (NIMS), in Daejeon, Korea in 2010 2012. Dr. Singh has earned two times fellowship award from APAN (Asia Pacific Advance Networks) meeting for Singapore, 2006 and Manila, 2007. He is serving as an Associate Editor of International Journal of Network Protocols and Algorithms for Smart Grids Protocols and Algorithms, USA. His research interests focus on the design, analysis, and implementation of algorithms and protocols for large-scale data set to solve realworld problems in the fields of internet oriented WSN applications, MANETs, 6lowpan, e-Healthcare system, wireless mesh network, scale free networks, IoT, machine-to-machine communication, future internet architecture \& services, etc. 